ELISpot would have fallen from 100\% to $92 \%$ and those with previously treated tuberculosis would have remained below the diagnostic threshold.

In this issue of Thorax, Breen et al ${ }^{12}$ take a slightly different approach using tuberculin testing and flow cytometry (see page 67). BAL fluid was taken from a substantially larger group of patients $(\mathrm{n}=250)$ in whom tuberculosis was suspected but the sputum smear was negative for auramine-positive bacilli, of whom 111 gained a diagnosis of active tuberculosis. The cells were first sorted for CD45 (common leucocyte antigen) and CD4 markers and 50000 of these cells were cultured with the mixture of antigens found in tuberculin-purified protein derivative (PPD). Flow cytometry was then used to measure the percentage of CD4+ cells which expressed interferon- $\gamma$ after stimulation with PPD. Although ESAT-6 was also used in 71 subjects, the specificity was not increased: all those who were positive with PPD but who did not have tuberculosis were also positive after stimulation with ESAT-6. The high falsepositive rating in this assay (24\%) was a significant problem. Breen et al suggest that many of these should have been treated for tuberculosis as 2 of 34 developed active disease within the period of follow-up. Several could well have had self-healed tuberculosis, as noted by the presence of apical shadowing and/or calcified mediastinal lymphadenopathy, and some had known contact with tuberculosis. The convenience of flow cytometry may have lost out to the specificity of the ELISpot.

Should we use the new tests for latent tuberculosis in the examination of BAL fluid? The answer remains unclear, but the excitement is palpable. Many questions remain. Might sputum induction provide a similar sensitivity? Can the ELISA-based tests perform as well as the ELISpot assays? At last the investment made during the last decade may begin to pay dividends in the clinical management of patients with suspected tuberculosis.

Funding: NHS Culyer allocation. Competing interests: None.

Thorax 2008;63:4-5. doi:10.1136/thx.2007.084202

\section{REFERENCES}

1. Rose AMC, Watson JM, Graham C, et al. Tuberculosis at the end of the 20th century in England and Wales: results of a national survey in 1998. Thorax 2001;56:173-9.

2. Watterson SA, Drobniewski FA. Modern laboratory diagnosis of tuberculosis. J Clin Pathol 2000;53:72732
3. Phillips M, Cataneo RN, Condos R, et al. Volatile biomarkers of tuberculosis in the breath. Tuberculosis 2007;87:44-52.

4. Daikos GL, Brooks JB, Michos A, et al. Detection of tuberculostearic acid in serum and other biological fluids from patients with tuberculosis by electroncapture gas chromatography and chemical ionization mass spectrometry. Int $\mathrm{J}$ Tuberc Lung Dis 2004:8:1027-31

5. Watanabe M, Aoyagi $Y$, Ridell $\mathbf{M}$, et al. Separation and characterization of individual mycolic acids in representative mycobacteria. Microbiology 2001;147:1825-37.

6. Condos R, Rom WN, Liu YM, et al. Local immune responses correlate with presentation and outcome in tuberculosis. Am J Respir Crit Care Med 1998; 157:729-35.

7. Dieli F, Sireci G, Ivanyi J, et al. Broad clonal heterogeneity of antigen-specific CD4+ T-cells localizing at the site of disease during tuberculosis. Immunol Letters 1999:69:311-5.

8. Tully G, Kortsik C, Höhn H, et al. Highly focused T cell responses in latent human pulmonary Mycobacterium tuberculosis infection. J Immunol 2005;174:2174-84

9. Sable SB, Goyal D, Verma I, et al. Lung and blood mononuclear cell responses of tuberculosis patients to mycobacterial proteins. Eur Respir J 2007;29:33746.

10. Jafari C, Eernst M, Kalsdorf B, et al. Rapid diagnosis of smear-negative tuberculosis by bronchoalveolar lavage enzyme-linked immunospot. Am J Respir Crit Care Med 2006;174:1048-54.

11. Arend SM, Thijsen SFT, Leyten EMS, et al. Comparison of two interferon- $\gamma$ assays and tuberculin skin test for tracing tuberculosis contacts. Am J Respir Crit Care Med 2007;175:618-27.

12. Breen RAM, Barry SM, Smith CJ, et al. Clinical application of a rapid lung-orientated immunoassay in individuals with possible tuberculosis. Thorax 2008;63:67-71.

\title{
Non-invasive ventilation for the treatment of hypercapnic respiratory failure in cystic fibrosis
}

\section{Peadar G Noone}

Median survival for patients with cystic fibrosis (CF) has improved steadily over the past several decades as a result of a multifaceted treatment approach to the disease. ${ }^{1}$ Despite this aggressive care, many patients with classic disease eventually develop respiratory failure from progressive airways obstruction and bronchiectasis. $^{2}$ At this stage, treatment

Correspondence to: Peadar G Noone, Pulmonary Division, CB\# 7020, UNC Chapel Hill, NC 27599-7020, USA; pnoone@med.unc.edu strategies may focus on alleviating both the symptomatic and physiological effects of respiratory failure. Initially respiratory failure may be mainly hypoxic (type 1: arterial oxygen pressure $\left(\mathrm{PaO}_{2}\right)<8 \mathrm{kPa}$ (or $60 \mathrm{~mm} \mathrm{Hg}$ ), with normal arterial carbon dioxide pressure $\left(\mathrm{PaCO}_{2}\right)$ ), and supplemental oxygen during sleep and/or exercise may be effective. However, when the disease becomes more severe, hypercapnia may ensue (type 2: $\mathrm{PaCO}_{2}>6 \mathrm{kPa}$ (or $45 \mathrm{~mm} \mathrm{Hg})$ ). Such patients with chronic respiratory failure usually have obvious clinical signs and symptoms, and blood gas analysis demonstrates a compensated respiratory acidosis. ${ }^{3}$ Thus it is important to periodically measure arterial blood gases in all patients with severe CF lung disease, to monitor for this serious complication of the disease.

When CF patients develop chronic respiratory failure, symptoms include the usual clinical sequelae of hypoxia and hypercapnia, as well as worsening dyspnoea associated with the increased work of breathing, sleep fragmentation and daytime fatigue, and reduced activities of daily living. Hypoxia with hypercapnia, and an elevation in serum bicarbonate, can support the need for adjunctive treatments. If oxygen treatment alone is judged insufficient, non-invasive ventilation (NIV) may be considered. ${ }^{3}$ Patients with CF may suffer acute respiratory failure during an exacerbation of moderate to severe disease, or indeed without any identified specific acute insult. A 2002 British Thoracic Society Standards of Care document listed the criteria for the use of NIV in acute respiratory failure. ${ }^{3}$ Of note, 
this document states that NIV should not be used routinely in CF and bronchiectasis because excessive secretions are likely to limit its effectiveness. Despite the dearth of randomised controlled trial data on the effectiveness of NIV in this situation, it is obvious from even a brief scan of the literature (and extensive anecdotal experience) that NIV is used frequently in CF patients with acute respiratory failure. ${ }^{4-7}$ While airway secretions might be a problem with NIV in CF, in practice this does not appear to be a major issue, and indeed there is some evidence that NIV (at least in single session treatments) augments clearance of airway secretions. ${ }^{8}$ Thus NIV has become a relied upon treatment modality in acute respiratory failure in CF, avoiding endotracheal intubation with its attendant complications in a substantial proportion of patients. ${ }^{6}{ }^{9}$ The situation is similar in chronic respiratory failure in CF. NIV use in CF was first described in the early 1990s, primarily in patients awaiting lung transplantation. 4910 The term "bridge" to transplantation was coined in reference to the observation that NIV could sustain life until more definitive life prolonging treatment could be offered, and this practice quickly became routine at centres capable of performing lung transplant for CF. ${ }^{4}$ Thus it is now common practice to employ NIV in chronic hypercapnic patients with CF, as a "bridge" to transplant, simply to sustain life outside the ICU or hospital setting, or to improve quality of life.

A 2003 comprehensive review of the state of the art of NIV in CF synthesised all of the available published data. ${ }^{11}$ It is clear from this document that the best randomised datasets supporting the use of NIV are single centre, hospital based, single session treatments, with small numbers of patients in each study. ${ }^{11}$ Specifically, three single session studies ( $n=62$ subjects) addressed the issue of airway clearance techniques, and lung function, and the data show modest improvements in airway clearance and some parameters of lung function. ${ }^{11}$ Two trials ( $\mathrm{n}=19$ subjects) evaluated NIV in a single overnight session in patients with CF, and showed that NIV offers benefits over either air or $\mathrm{O}_{2}$, by maintaining tidal volume and improving oxygenation without adding to hypercapnia. ${ }^{11}$ The conclusions of this detailed review were still rather stark-there was modest evidence to support the use of NIV in patients with $\mathrm{CF}$, and there were no long term data on its safety or efficacy. The conclusion called for a long term, multicentre randomised study in CF, to assess its efficacy in assisting airway clearance, and as a ventilation mode.

So, has that "perfect" study arrived? Not yet, but this issue of Thorax does contain a report by Young and colleagues $^{12}$ that goes some way towards filling the gap (see page 72). This Australian study looks at the utility of NIV over a longer time period ( 6 weeks) in a randomised, placebo controlled, crossover study design, comparing nocturnal NIV to low flow oxygen, and to air (as placebo). Although the final numbers were small ( $n=8$, with seven completing the NIV arm), reflecting the difficulty in recruiting and retaining subjects for these laborious types of studies even in a large CF centre, the study was well designed and executed. The CF centre, in Melbourne, Australia, has $\sim 250$ adults with CF, and despite screening 60 patients, only 14 fitted the inclusion criteria, with nine consenting and eight entering the study. The authors discuss the reasons for these limitationsfor example, the time commitment required of the subjects and the discomfort of the rather invasive tests required for completion, all reasonable issues for a group of rather ill patients. Inclusion criteria were clinical stability with at least mild obstructive lung disease (in practice, all patients who completed the study had severe disease), positive sleep studies (hypoxia at night) and daytime hypercapnia (>45 mm Hg). Nocturnal NIV was delivered via a nasal or face mask, with great attention to detail in regard to patient education and compliance, and appropriate adjustment of inspiratory and expiratory pressures according to $\mathrm{PCO}_{2}$ and/or obstructive events. The comparison treatment consisted of nocturnal nasal oxygen titrated to correct oxyhaemoglobin desaturation ( $>90 \%$ ), and the placebo was air delivered via a sham $\mathrm{O}_{2}$ delivery system similar to the actual $\mathrm{O}_{2}$ delivery. Subjects completed the relevant 6 weeks of intervention, followed by a 2 week washout period, and then switched randomly to one of the remaining two interventions, and so on, until all subjects had completed all three interventions. Key outcome measures were quality of life measures, daytime sleepiness and dyspnoea. Secondary outcomes included sleep architecture, awake and asleep blood gases, pulmonary function tests, neurocognitive tests and shuttle tests.

So, after this rather labour intensive study, what are the results? Demographics first. The group studied were mostly older (mean age 37 years), male, reasonably well nourished (mean body mass index $21.1 \mathrm{~kg} / \mathrm{m}^{2}$ ) with, as might be expected, severe lung disease (forced expiratory volume in $1 \mathrm{~s} 35 \%$ predicted on average). None was previously on home oxygen or had prior treatment with NIV. Interestingly, the five who fitted the inclusion criteria, but declined the study, were quite a bit younger (mean age 24 years), had similar lung function but were less well nourished. Most (7/8) tolerated the NIV well at pressures of $\sim 12 / 5 \mathrm{~cm} \mathrm{H}_{2} \mathrm{O}$, although total usage per night was $4.3 \mathrm{~h}$. The outcome data confirmed the study hypothesis that NIV is superior to low flow $\mathrm{O}_{2}$ or placebo, in this fairly sick group of older CF patients - chest symptoms scores and dyspnoea were better after NIV (table 2), although other quality of life measures showed no changes. Although NIV was generally well tolerated with almost no adverse effects, oxygen was the preferred treatment for most subjects (4/7 of those finishing all arms of the study). NIV improved nocturnal (but not daytime) hypercapnia measures. Finally, shuttle tests improved by $83 \mathrm{~m}$ on average, with several patients improving distances by over $50 \mathrm{~m}$ compared with air (on average, shuttle distances dropped on air and oxygen alone). Adverse effects included one pneumothorax, but this occurred on low flow air, and was likely a coincidence.

This paper is a very well constructed study of NIV in CF, although at first glance the numbers seem small and the benefits may seem marginal. Its significance lies in the fact that it is the first randomised, placebo controlled study of NIV over a reasonably long time in CF adults in respiratory failure. As any clinician who deals with this group of patients will recognise, and as noted above, NIV has been used for some time in these ill patients (well documented in the literature for many years ${ }^{4}$ ), and the benefits shown in this study are welcome-that is, the improved nocturnal ventilation, symptoms and exercise capacity data. Some patients can be maintained for considerable lengths of time on NIV-for example, during the wait time for lung transplantation. Some patients may decline lung transplantation, or be ineligible for various reasons, yet derive considerable symptomatic and physiological benefit from NIV for long periods of time. It is worth noting that the duration of this study involved 6 week treatment arms, while in practice many patients use NIV for much longer periods of time; thus, the benefits gained may be even greater than those shown in this study. 
Another point of note is that NIV use in all patients (CF and non-CF) with respiratory failure has a significant impact on the Lung Allocation Score, a system currently in use in the USA to prioritise the utility of donor lungs for all waiting recipients; thus, those with higher scores (such as CF patients using chronic NIV) gain higher priority for organ acquisition while on the transplant wait list. ${ }^{13}$

Finally, in the era of evidence based medicine, and third party payer expectations of such data, it is useful to have peer reviewed data from studies such as this, to support the use of this treatment modality. In practice, as the authors declare at the conclusion of their paper, only by performing multicentre studies of NIV in CF with larger numbers and perhaps longer study times will it be possible to improve and expand on the findings of this study.
Acknowledgements: The author gratefully acknowledges the helpful comments of James $\mathrm{R}$ Yankaskas during the writing of this manuscript.

Competing interests: None.

Thorax 2008:63:5-7. doi:10.1136/thx.2007.086710

\section{REFERENCES}

1. CFF. 2006. Cystic Fibrosis Foundation, Patient Registry 2005 Annual Report. Cystic Fibrosis Foundation Annual Data Report. Bethesda, MD: Cystic Fibrosis Foundation, 2005:2-3.

2. Gibson RL, Burns JL, Ramsey BW. Pathophysiology and management of pulmonary infections in cystic fibrosis. Am J Respir Crit Care Med 2003;168:91851.

3. British Thoracic Society. Non-invasive ventilation in acute respiratory failure. Thorax 2002;57:192-211.

4. Hodson ME, Madden BP, Steven MH, et al. Noninvasive mechanical ventilation for cystic fibrosis patients - a potential bridge to transplantation. Eur Respir J 1991;4:524-7.

5. Piper AJ, Parker S, Torzillo PJ, et al. Nocturnal nasal IPPV stabilizes patients with cystic fibrosis and hypercapnic respiratory failure. Chest 1992;102:846-50.

6. Sood N, Paradowski LJ, Yankaskas JR. Outcomes of intensive care unit care in adults with cystic fibrosis. Am J Respir Crit Care Med 2001;163:335-8.
7. Vedam H, Moriarty C, Torillo PJ, et al. Improved outcomes of patients with cystic fibrosis admitted to the intensive care unit. J Cyst Fibros 2004;3:8-14.

8. Bradley JM, Moran FM, Elborn JS. Evidence for physical therapies (airway clearance and physical training) in cystic fibrosis: an overview of five Cochrane systematic reviews. Respir Med 2006;100:191-201.

9. Hill AT, Edenborough FP, Cayton RM, et al. Longterm nasal intermittent positive pressure ventilation in patients with cystic fibrosis and hypercapnic respiratory failure (1991-1996). Respir Med 1998:92:523-6.

10. Madden BP, Kariyawasam H, Siddiqi AJ, et al. Noninvasive ventilation in cystic fibrosis patients with acute or chronic respiratory failure. Eur Respir $J$ 2002;19:310-13

11. Moran F, Bradley J. Non-invasive ventilation for cystic fibrosis. Cochrane Database Syst Rev 2003;CD002769. Chichester: Wiley InterScience, 2003

12. Young AC, Wilson JW, Kotsimbos TC, et al. Randomised placebo controlled trial of non-invasive ventilation for hypercapnia in cystic fibrosis. Thorax 2008; 63:72-7.

13. Coke M, Edwards LB. Current status of thoracic organ transplantation and allocation in the United States. Clin Transp/ 2004:17-26.

\section{Lung alert}

\section{CT and lung function patterns in bronchopulmonary dysplasia (BPD) in the post-surfactant era}

Advances in neonatal management, particularly the use of surfactant, have been associated with the emergence of a new form of bronchopulmonary dysplasia (BPD). This is thought to be a result of arrested lung development and is characterised by abnormalities of alveolar septation and microvascular maturation. This retrospective review describes chest computerised tomography (CT) and lung function patterns in 41 very low birth weight babies with symptomatic BPD who received optimal treatment including surfactant. These infants underwent CT scanning and lung function testing (VmaxFRC and FRC (functional residual capacity)) 10-20 months after birth for persistent respiratory symptoms.

All CT scans were abnormal, the most frequent patterns being hyperlucent areas (88\%), linear opacities (94\%), triangular subpleural opacities $(71 \%)$ and bullae $(53 \%)$. The number of subpleural and linear opacities, but not hyperlucency, correlated negatively with FRC and positively with duration of oxygen exposure, suggesting different mechanisms for these changes.

This study highlights the changing CT patterns in BPD, reflecting differences in management as well as pathogenesis. The association of CT opacities with low FRC suggests persistent pulmonary fibrotic lesions despite newer therapies. Several CT features are similar to those seen in the pre-surfactant era, with the notable absence of bronchiectasis, and remain associated with the duration of oxygen therapy and mechanical ventilation.

- Mahut B, de Blic J, Emond S, et al. Chest computed tomography findings in bronchopulmonary dysplasia and correlation with lung function. Arch Dis Child Fetal Neonatal Ed 2007;92:F459-64

\section{A Krishnan}

Correspondence to: A Krishnan, Clinical Research Fellow, Thoracic Medicine, St Vincent's Hospital, Sydney, Australia; akrishnan@stvincents.com.au 